\title{
Correlation-driven charge migration following double ionization and attosecond transient absorption spectroscopy
}

\author{
Maximilian Hollstein, ${ }^{1,2}$ Robin Santra, ${ }^{3,1,2}$ and Daniela Pfannkuche ${ }^{1,2}$ \\ ${ }^{1}$ Department of Physics, University of Hamburg, Jungiusstraße 9, D-20355 Hamburg, Germany \\ ${ }^{2}$ The Hamburg Centre for Ultrafast Imaging, Luruper Chaussee 149, 22761 Hamburg, Germany \\ ${ }^{3}$ Center for Free-Electron Laser Science, DESY, Notkestraße 85, 22607 Hamburg, Germany
}

(Received 20 May 2016; published 22 May 2017)

\begin{abstract}
We theoretically investigate charge migration following prompt double ionization. Thereby, we extend the concept of correlation-driven charge migration, which was introduced by Cederbaum and coworkers for single ionization [Chem. Phys. Lett. 307, 205 (1999)], to doubly ionized molecules. This allows us to demonstrate that compared to singly ionized molecules, in multiply ionized molecules, electron dynamics originating from electronic relaxation and correlation are particularly prominent. In addition, we also discuss how these correlationdriven electron dynamics might be evidenced and traced experimentally using attosecond transient absorption spectroscopy. For this purpose, we determine the time-resolved absorption cross section and find that the correlated electron dynamics discussed are reflected in it with exceptionally great detail. Strikingly, we find that features in the cross section can be traced back to electron hole populations and time-dependent partial charges and hence, can be interpreted with surprising ease. By taking advantage of element-specific core-to-valence transitions even atomic spatial resolution can be achieved. Thus, with the theoretical considerations presented, not only do we predict particularly diverse and correlated electron dynamics in molecules to follow prompt multiple ionization but we also identify a promising route towards their experimental investigation.
\end{abstract}

DOI: 10.1103/PhysRevA.95.053411

\section{INTRODUCTION}

The dynamics of electrons govern elementary processes such as chemical reactions and charge transport in molecules, biological systems, and solid-state samples. The observation and understanding of these processes is the central goal of ultrafast science. With the advent of attosecond pulses, it is becoming possible to steer and to probe these electron dynamics on their natural timescale $[1,2]$. In this context, of particular interest is charge migration which represents a potentially ultrafast and efficient first step of a charge transfer which can occur even prior to the onset of a significant nuclear motion. Charge migration in molecules can take place whenever a coherent superposition of multiple electronic states is prepared. So far, this has been achieved by ionization with attosecond pulses [3] and by strong-field ionization [4] whereby the dynamics triggered could be well understood on the basis of a single active particle picture. Still an outstanding issue of attosecond physics is correlation-driven charge migration [5,6] representing a charge transfer mechanism that occurs solely due to electron correlation and orbital relaxation. The experimental demonstration of the process, however, is still lacking.

In this context, we address two unresolved aspects: First, we present a so far outstanding extension of the concept of correlation-driven charge migration [5] towards multiply ionized molecules. This allows us to demonstrate the particular prominence of a specific class of correlation-driven processes that can follow the emission of multiple electrons but which are absent in singly ionized closed-shell molecules. These pure electron dynamics discussed can occur in many situations in which multiple electrons are promptly removed from a molecule, i.e., when the electron removal occurs during a time span that is short compared to the dynamical timescales associated with valence excitations. Such situations can be the consequence of attosecond ionization which can be induced by either attosecond light pulses or by strong-field ionization [7-9]. Importantly, prompt removal of multiple electrons can also be the result of electronic decay processes that follow for instance inner-shell ionization. Core-hole lifetimes associated with secondary electron emission are usually on the order of a few femtoseconds and can be as short as a few hundred attoseconds. Therefore, also secondary electron emission in the course of an electronic decay can represent a prompt double ionization event which will be ensued by the kind of electron dynamics that are discussed in this work. The second aspect discussed concerns the experimental tracing of such often rather involved electron dynamics that are due to electron correlation and relaxation. In light of the availability of attosecond pulses, the detection and experimental investigation of correlation-driven electron dynamics can be anticipated for the near future. However, even for simple systems, the interpretation of attosecond experiments is usually complicated [10]. Therefore, the tracing of correlated electron dynamics in molecules appears currently still an outstanding problem. Here, we address this important issue constructively by presenting theoretical predictions concerning attosecond transient absorption spectroscopy (ATAS) applied to correlated electron dynamics in a molecule. With this, we provide evidence that ATAS is particularly suitable for this objective.

Among those techniques which have been proposed for monitoring ultrafast electron dynamics [11-16], ATAS [7,1719] stands out since it combines high spectral and high temporal resolution. So far, it has already been successfully applied to the electron dynamics in atoms [7,17,20-23] as well as to the dynamics in solid state systems [24,25]. An application to correlated electron dynamics in polyatomic molecules, however, has not yet been conducted and it has not been clear to which extent ATAS can be used to gain insight into correlation-driven dynamics which can be rather complex involving very many electronic states. 
Here, we demonstrate that when spectrally resolved, the time-resolved absorbance allows surprisingly intuitive and highly detailed insight into correlated electron dynamics in molecules. Concretely, we demonstrate that features in the ATA spectra can be directly related to quantities such as partial charges and orbital populations so that intuitive insight into the valence electron dynamics can be obtained. Remarkably, we find that ATAS allows one to differentiate dynamics that originate from different excitation classes and thus offers exceptionally detailed insight. Moreover, when the element specificity of core-to-valence transitions can be exploited, even atomic spatial resolution can be achieved. Hence, for molecules for which this is possible, charge transfer processes can be traced in real space.

These very promising results concerning ATAS applied to involved electron dynamics in molecules, we identify a promising route for future experiments and theoretical studies aiming at the real-time dynamics of ultrafast charge transfer processes triggered by electron correlation as well as nuclear motion [26].

\section{CORRELATION-DRIVEN CHARGE MIGRATION FOLLOWING DOUBLE-IONIZATION}

Charge migration following ionization can be triggered by short ionizing light pulses, which prepare the ion in a coherent superposition of several electronic states. For closedshell molecules, which represent the vast majority of stable molecules, the ground state can usually be well approximated by the Hartree-Fock determinant so that ionization caused by a perturbation that is represented by a one-particle operator such as light, predominantly prepares a superposition of one-hole $(1 \mathrm{H})$ configurations, i.e., states that result from the annihilation of an electron from a Hartree-Fock orbital from the Hartree-Fock ground-state determinant.

Notably, those $1 \mathrm{H}$ configurations that represent states, in which the hole is in an outer-valence orbital, are usually excellent approximations to exact eigenstates of the ionic Hamiltonian. This is due to the fact that $1 \mathrm{H}$ configurations are not directly coupled with each other and that situations where outer-valence $1 \mathrm{H}$ configurations are significantly coupled to higher excitation classes [27,28] are rather the exception than the rule [6,29-33]. As a consequence, outer-valence single ionization of closed-shell molecules typically initiates dynamics of a single (quasi-) particle, i.e., a single electron hole.

Inner-valence $1 \mathrm{H}$ configurations, however, are usually significantly coupled by the residual electron-electron interaction, i.e., the difference of the mean-field potential associated with the Hartree-Fock ground state and the bare Coulomb interaction, to the two-hole one-particle (2H1P) configurations [6,29-33] so that inner-valence ionization can be followed by dynamics involving more than a single particle, which can usually not be described by single-determinant approaches.

In this work, we extend this concept of correlation-driven charge migration to the dynamics that follow double ionization. Therefore, we consider the field-free time evolution of two-hole configurations, i.e., states that result from the annihilation of two electrons from Hartree-Fock orbitals from the Hartree-Fock ground state. The electron dynamics that follow the preparation of such a state are solely due to the residual electron-electron interaction. It is important to note that since the Schrödinger equation is a linear equation, the mechanisms described are still operating when an ionization process (e.g., two-photon absorption or secondary electron emission associated with a molecular Auger decay) prepares a superposition of $2 \mathrm{H}$ configurations. Therefore, the conclusions drawn from our calculations are not exclusively valid for the initial states considered, but they apply to coherent electron dynamics following double ionization in general. Importantly, in contrast to $1 \mathrm{H}$ configurations, the residual electron-electron interaction couples $2 \mathrm{H}$ configurations directly. Namely, the off-diagonal Hamiltonian matrix elements of $2 \mathrm{H}$ configurations are given by Coulomb integrals that can be identified as the repulsive Coulomb interaction of two electron holes.

Note that these kinds of processes, which arise from this hole-hole interaction, are specific to multiply ionized systems; in singly ionized closed-shell molecules, they are absent. It is important to note that they give rise to a particular prominence of correlation-driven electron dynamics in multiply ionized molecules. As demonstrated in the following, this is manifested by the fact that the prompt removal of multiple electrons from outer-valence orbitals can initiate diverse and correlated electron dynamics. As in singly ionized molecules, the residual electron-electron interaction can also cause the promotion of electrons to initially unoccupied orbitals giving rise to additional dynamics. For low ionization energies, this is predominantly due to the coupling of the $2 \mathrm{H}$ configurations to the three-hole-one-particle $(3 \mathrm{H} 1 \mathrm{P})$ configurations. To provide a transparent picture of the diverse dynamics that are driven by the residual electron-electron interaction, we perform two kinds of calculations-one concerning the dynamics that are solely driven by the hole-hole repulsion. For this purpose, the time-dependent electronic wave function is expanded in terms of $2 \mathrm{H}$ configurations:

$$
|\psi(t)\rangle=\sum_{i<j} \alpha_{i, j}(t) c_{i} c_{j}\left|\Phi_{0}\right\rangle .
$$

Here, $c_{i}, c_{j}$ denote annihilation operators of Hartree-Fock spin orbitals and $\left|\Phi_{0}\right\rangle$ denotes the Hartree-Fock ground state of the neutral closed-shell molecule. In a second kind of calculation, the promotion of an electron to initially unoccupied orbitals is incorporated. For this purpose, we expand the wave function in terms of the $2 \mathrm{H}$ configurations and the $3 \mathrm{H} 1 \mathrm{P}$ configurations:

$$
\begin{aligned}
|\psi(t)\rangle= & \sum_{i<j} \alpha_{i, j}(t) c_{i} c_{j}\left|\Phi_{0}\right\rangle \\
& +\sum_{a} \sum_{i<j<k} \alpha_{i, j, k}^{a}(t) c_{a}^{\dagger} c_{i} c_{j} c_{k}\left|\Phi_{0}\right\rangle .
\end{aligned}
$$

The propagation in time of the initial states considered was performed with the exact field-free Hamiltonian restricted to the respective configuration space. Details concerning the numerical treatment are described in the Appendix.

\section{ATTOSECOND TRANSIENT ABSORPTION SPECTROSCOPY}

ATAS is based on the transmission of a spectrally broadband attosecond probe pulse with central photon energies suitable 
for the excitation of an electron from an inner shell to the valence shell. After the transmission through the sample under investigation, the pulse is spectrally dispersed and analyzed. This allows one to monitor the valence electron dynamics with both high temporal and high spectral resolution. When applied to molecules, strong atomic localization of the inner-shell orbitals and element-specific inner-shell orbital energies make atomic resolution possible [16,34]. In the work presented, the theoretical ATA spectra are obtained by following the procedure given in Ref. [35] in the short-pulse approximation (i.e., the probe pulse is assumed to be a $\delta$ pulse). Assuming unaligned molecules, we consider an orientation-averaged cross section. Details concerning the numerical treatment are given in the Appendix. As a specific example, we consider the $\mathrm{C}_{2} \mathrm{H}_{4} \mathrm{BrI}$ molecule. Since the iodine- $4 d$ to valence transitions and the bromine- $3 d$ to valence transitions are energetically well separated, ATAS allows for this molecule insight into the valence electron dynamics with atomic spatial resolution. That is, the dynamics that occur locally at the iodine and bromine atoms, respectively, can be resolved. In the ATA spectra presented in the following, features that occur at photon energies in the region from 46-67 eV involve only final states that are associated with the promotion of an electron from an iodine- $4 d$-type orbital to the valence shell. As in Ref. [36] where a similar molecule is subject to core-tovalence spectroscopy, we refer to this spectral region as the iodine window. For the same reason, the region of $67-80 \mathrm{eV}$ is referred to as the bromine window, since there only transitions to final states are relevant that are associated with the promotion of an electron from the bromine-3d-type inner shell to the valence shell.

\section{SPECIFIC EXAMPLES}

In the following, aspects regarding correlation-driven dynamics in doubly ionized molecules and their manifestation in the time-resolved absorption cross section are exemplified considering correlation-driven processes in the $\mathrm{C}_{2} \mathrm{H}_{4} \mathrm{BrI}$ dication.

\section{A. Outer-valence double ionization}

First, we consider electronic wave packets produced via outer-valence double ionization. Specifically, the situation is considered where two electrons are suddenly removed from the highest occupied molecular orbital (HOMO) of $\mathrm{C}_{2} \mathrm{H}_{4} \mathrm{BrI}$. In the calculations artificially restricted to the two-hole configuration subspace, the resulting dynamics are governed by the coherent superposition of two dicationic eigenstates (see Fig. 1):

$$
\left|\Psi_{2 H}(t)\right\rangle=0.74 \times e^{-i E_{1} t}\left|\Psi_{1}\right\rangle+0.57 \times e^{-i E_{2} t}\left|\Psi_{2}\right\rangle \ldots
$$

with $E_{1}-E_{2}=1.58 \mathrm{eV}$. Both states are superpositions of mostly two spin-adapted singlet $2 \mathrm{H}$ configurations:

$$
\begin{aligned}
\left|\Psi_{1}\right\rangle= & 0.74 \times|\mathrm{HOMO}, \mathrm{HOMO}\rangle \\
& +0.51 \times|\mathrm{HOMO}, \mathrm{HOMO}-6\rangle \ldots \\
\left|\Psi_{2}\right\rangle= & 0.57 \times|\mathrm{HOMO}, \mathrm{HOMO}\rangle \\
& -0.69 \times|\mathrm{HOMO}, \mathrm{HOMO}-6\rangle \ldots
\end{aligned}
$$

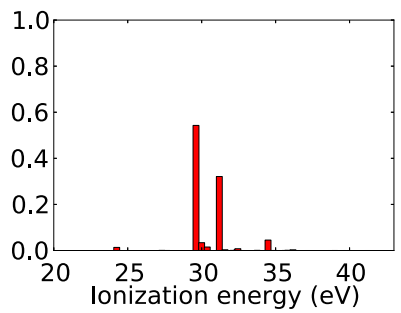

(a) $2 \mathrm{~h}$

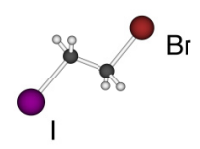

(c) $\mathrm{C}_{2} \mathrm{H}_{4} \mathrm{BrI}$ (b) $2 \mathrm{~h}+3 \mathrm{~h} 1 \mathrm{p}$

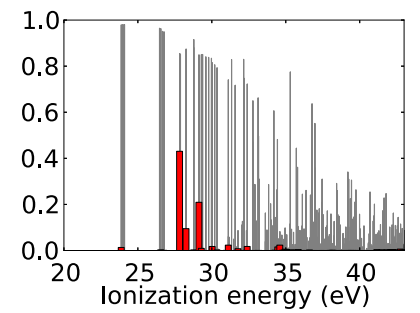

FIG. 1. (a), (b) Spectral decomposition (thick, red bars) of the $2 \mathrm{H}$ configuration, in which both holes are in the HOMO orbital, within the respective approximation. (a) Only the subspace spanned by the $2 \mathrm{H}$ configurations is considered. (b) The $3 \mathrm{H} 1 \mathrm{P}$ configurations are included in the calculation. Thin, gray bars represent the weight of $2 \mathrm{H}$ configurations in the dicationic eigenstates. (d), (e) Isosurfaces of the molecular orbitals most prominently involved in the dynamics.

That is, the dynamics are associated with oscillatory hole populations of the HOMO and the HOMO-6. Noting that the HOMO is primarily localized at the iodine atom whereas the HOMO-6 is mostly localized at the $\mathrm{C}_{2} \mathrm{H}_{4}$ group [see Fig. 1(e)], the dynamics correspond to an oscillation of a hole between the iodine atom and $\mathrm{C}_{2} \mathrm{H}_{4}$ group with a period of $\sim 2.6 \mathrm{fs}$. This charge oscillation driven by the hole-hole interaction is reflected in the time-resolved absorption cross section in the iodine window [see Fig. 2(a)]. In fact, integration of the cross section over the iodine window $(\sim 46-67 \mathrm{eV})$ yields a quantity that is proportional to the partial charge of the iodine atom (see Fig. 3). In the bromine window ( $67-80 \mathrm{eV})$, however, no signal is observable, reflecting the fact that the charge dynamics are restricted to the iodine atom and the $\mathrm{C}_{2} \mathrm{H}_{4}$ group.

Remarkably, when including particle-hole excitations in the calculation, it becomes apparent that even though the electrons are removed from the outer-valence shell where these processes are typically assumed to be of minor importance [6,29-33], they noticeably affect the dynamics [see Fig. 1(b) and Fig. 2(b)] and give rise to prominent additional features in the time-resolved absorption cross section. Besides the dominant feature at $\sim 53 \mathrm{eV}$, which is already present when the calculations are restricted to the $2 \mathrm{H}$ subspace, at $\sim 61 \mathrm{eV}$ and $\sim 77 \mathrm{eV}$ new features arise [see Fig. 2(b)]. The feature at $61 \mathrm{eV}$ is within the iodine window $(\sim 46-67 \mathrm{eV})$ and therefore can be related to charge dynamics at the iodine atom. As the feature at $\sim 53 \mathrm{eV}$, it is a result of the coherent population of the two most prominently populated states.

In contrast to the feature at $\sim 53 \mathrm{eV}$, which reflects the hole dynamics that originate from the hole-hole repulsion, however, it monitors the charge dynamics that are associated with the $3 \mathrm{H} 1 \mathrm{P}$ component of the wave function. This is indicated by the fact that the final states involved in the feature are predominantly constituted by $3 \mathrm{H} 1 \mathrm{P}$ configurations (i.e., 


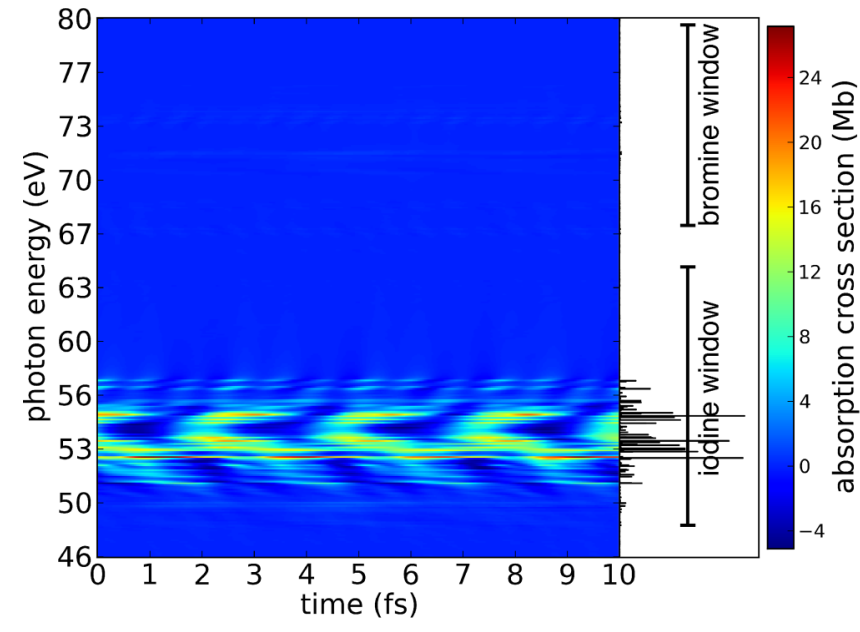

(a)

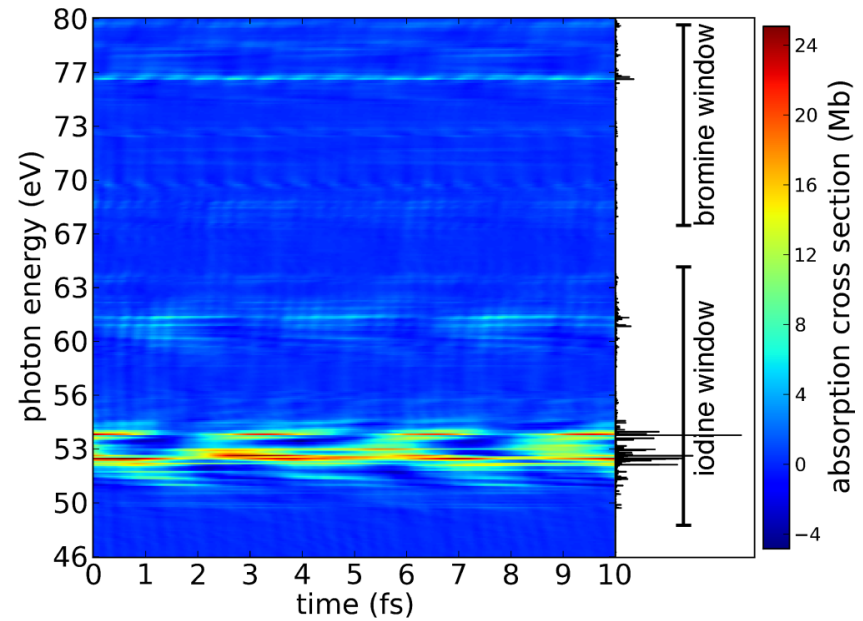

(b)

FIG. 2. The time-resolved absorption cross section for the situation where at $t=0$, the $2 \mathrm{H}$ configuration is prepared in which both holes are in the HOMO. (a) In the calculation, only the subspace spanned by the $2 \mathrm{H}$ configurations is taken into account. (b) $3 \mathrm{H} 1 \mathrm{P}$ configurations are included in the calculation.

the weight of $3 \mathrm{H} 1 \mathrm{P}$ configurations in these final states is larger than $\geqslant 0.9$ ). Moreover, it reflects the time-dependence of the iodine partial charge that is obtained from the 3H1P component of the wave function (see Fig. 4). The feature in the bromine window at $\sim 77 \mathrm{eV}$ can be related to a coherent superposition of the most prominently populated state with a double ionization potential (DIP) of $27.7 \mathrm{eV}$ and a group of dicationic states with dominant $3 \mathrm{H} 1 \mathrm{P}$ character (i.e., the weight of $3 \mathrm{H} 1 \mathrm{P}$ configurations in these states is larger than 0.9 ) and DIPs on the order of $\sim 34.5 \mathrm{eV}$. The associated energy difference of $6.7 \mathrm{eV}$ is manifested in the oscillation of the cross section with a period of $\sim 610$ as. Hence, this signal at $77 \mathrm{eV}$ is caused by the charge dynamics that occur locally at the bromine atom resulting from particle-hole excitations.

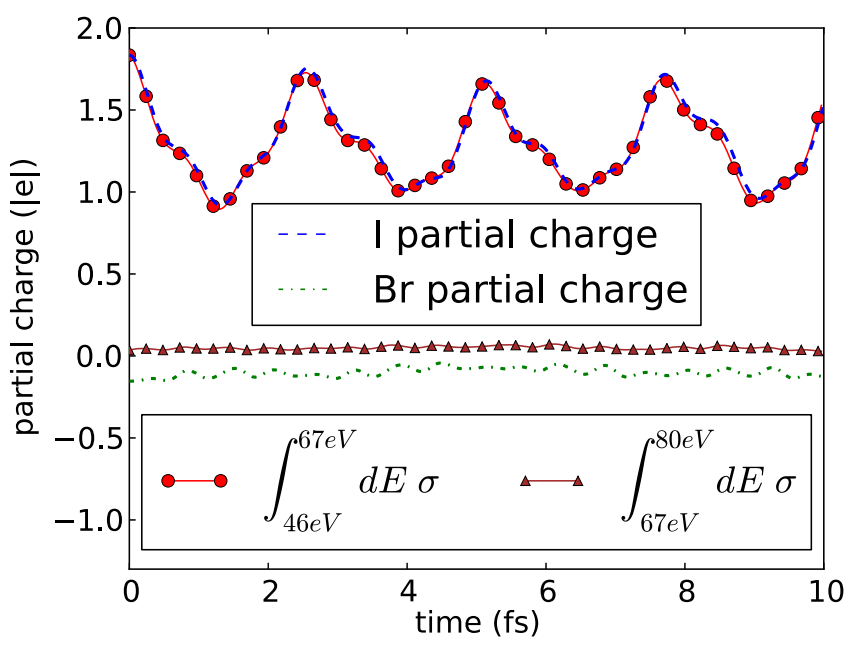

FIG. 3. The time-dependent partial charges (dashed lines) obtained by Löwdin population analysis [37] in units of the elementary positive charge and the cross section $\sigma$ in arbitrary units integrated over selected spectral regions (solid lines with markers) perfectly reflecting the hole charge dynamics (see text).

\section{B. Inner-valence double ionization}

When electrons are emitted from inner-valence orbitals, particle-hole excitations dominate the subsequent electron dynamics in the ionic system. For single ionization, this situation has been termed "breakdown of the molecular orbital picture of ionization" $[38,39]$, which is characterized by the population of many ionic eigenstates with rather low $1 \mathrm{H}$ weight. In the following, we consider the analogous situation for double ionization (see, for instance, Ref. [40]) realized by a sudden removal of two electrons from the HOMO-5 [see Fig. 5(a)]. The hole population dynamics [see Fig. 5(b)] that

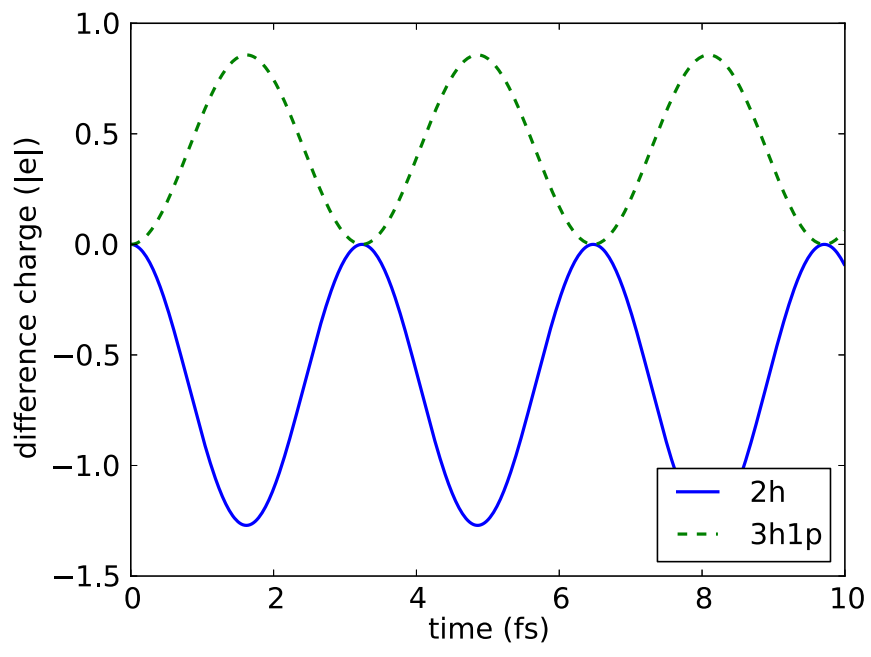

FIG. 4. The time-dependent difference iodine partial charge $\Delta Q$ [ $\Delta Q=Q(t)-Q(t=0) ; Q$ denotes here the iodine partial charge determined by Löwdin population analysis] obtained from the $3 \mathrm{H} 1 \mathrm{P}$ component (dashed, green curve) and, respectively, the $2 \mathrm{H}$ component (solid, blue curve) of the coherent superposition of the two dicationic eigenstates that are predominantly populated when the $2 \mathrm{H}$ configuration is prepared in which both holes are in the HOMO-5 orbital. 


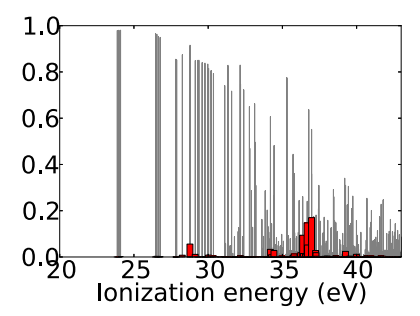

(a)
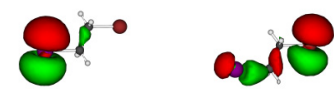

(c) HOMO-1

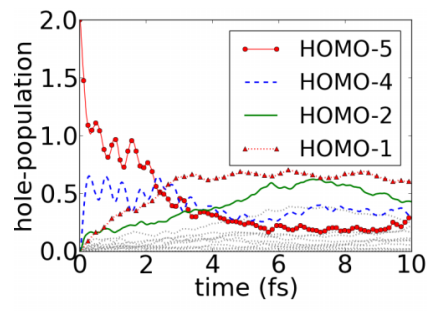

(b)
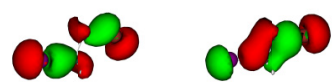

(f) HOMO-5
FIG. 5. (a) Spectral decomposition (thick, red bars) of the spinadapted two-hole configuration in which both electron holes are in the HOMO-5 orbital (|HOMO - 5,HOMO-5 $\rangle$ ) and the weight of the $2 \mathrm{H}$ configurations in the dicationic singlet eigenstates (thin, gray bars). (b) The time-dependent populations of Hartree-Fock orbitals by electron holes following the preparation of the state $|\mathrm{HOMO}-5, \mathrm{HOMO}-5\rangle$. (c)-(f) The isosurfaces of the molecular orbitals most prominently populated by electron holes in the course of these dynamics, cf. (b).

follow the preparation exhibit a repopulation of the HOMO-5 by an electron, an according depopulation of the HOMO-1 and HOMO-2 on a few-fs timescale ( $\sim 5 \mathrm{fs})$ and a very fast depopulation of the HOMO-4 within only 300 as. These dynamics can be observed in the time-resolved absorption cross section (see Fig. 6). The HOMO-5 is delocalized throughout the whole molecule [see Fig. 5(f)] so that its depopulation can be observed at photon energies in both the iodine window at $\sim 56 \mathrm{eV}$ and the bromine window at $\sim 72 \mathrm{eV}$. It turns out that the cross section integrated from $55-60 \mathrm{eV}$ and from 70-72 eV, respectively, shows a similar trend as the hole population of the HOMO-5. However, as shown

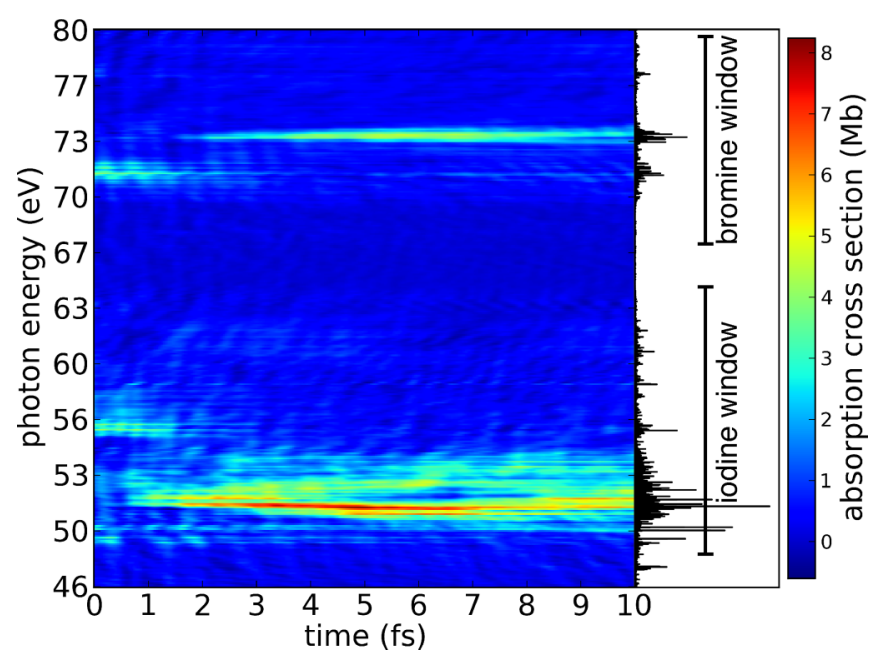

FIG. 6. The time-resolved absorption cross section for the situation where at $t=0$, the two-hole configuration is prepared in which both holes are in the HOMO-5. Particle-hole excitations are taken into account. Black bars at the right side of the panel reflect the weight of the transitions involved.

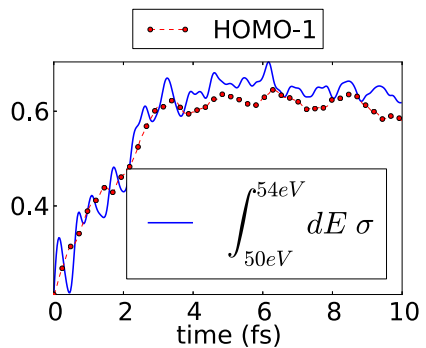

(a)

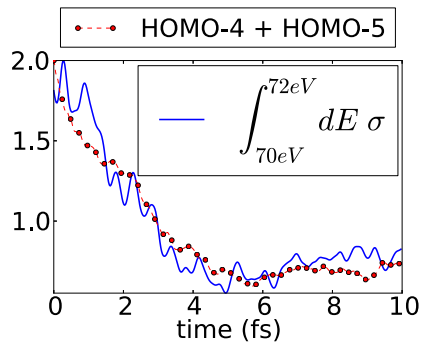

(c)

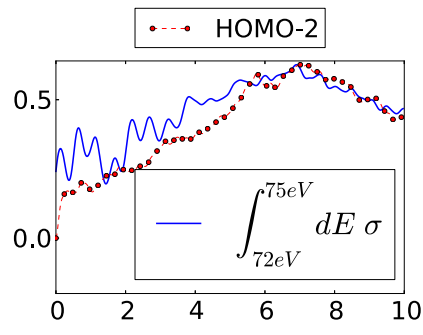

(b)

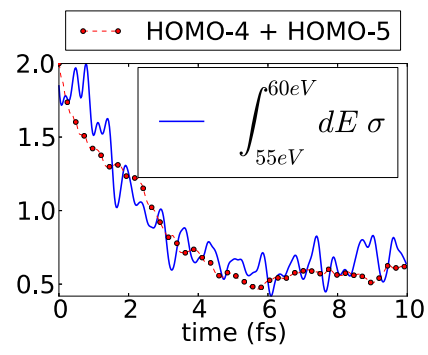

(d)
FIG. 7. The time-resolved absorption cross section $\sigma$ in arbitrary units (see Fig. 6) integrated over selected spectral regions (blue, solid curve) and time-dependent hole populations (red, dotted curves) for the situation where at $t=0$, the two-hole configuration is prepared in which both holes are in the HOMO-5. The time-dependent populations of Hartree-Fock orbitals by electron holes are reflected in the piecewise integrated absorption cross section (see text).

in Fig. 7(c) and Fig. 7(d) excellent agreement is obtained in both cases rather with the sum of the hole populations of the HOMO-5 and the HOMO-4 and not solely the hole population of the HOMO-5. This can be attributed to the very similar character of these two orbitals. That is, both orbitals are completely delocalized throughout the whole molecule so that both orbitals have large overlap with both the bromine and the iodine atom.

In contrast, the HOMO- 1 and the HOMO-2 can be distinguished since the HOMO-1 is almost exclusively localized at the iodine atom whereas the HOMO-2 is localized at the bromine atom. Their populations are correlated with the features in the ATA spectrum at $\sim 52 \mathrm{eV}$ and at $\sim 73 \mathrm{eV}$, respectively. The very prominent population of the HOMO-1 is proportional to the absorption cross section integrated over the very prominent feature at $50-54 \mathrm{eV}$ whereas the population of the HOMO-2 is proportional to the cross section integrated from 72-75 eV (see Fig. 7).

Remarkably, while the complicated substructure of the feature at $\sim 50-54 \mathrm{eV}$ reflects the involvement of many dicationic eigenstates and rather complicated electron dynamics, the hole dynamics can rather simply be extracted from the ATA spectra. In this context, it should be noted that in an experiment, the substructure is likely to be subject to broadening due to nuclear motion [41]; information about the hole dynamics, however, might be still obtained by integration over the feature.

Notably, features associated with the emission of radiation (i.e., features where the absorption cross section is negative) known from atomic ATA spectra [35] are missing. This appears to be related to the involvement of very many transitions with only slightly different transition energies. The overlap of these 
many lifetime-broadened transition lines apparently causes a primarily positive cross section.

To conclude, we have demonstrated that in contrast to single ionization, for double ionized molecules, correlation-driven charge migration is particularly prominent, and exceptionally rich electron dynamics can already follow outer-valence double ionization. By showing that these dynamics can be well monitored by ATAS, we have also presented theoretical predictions concerning ATAS applied to the correlated electron dynamics in a polyatomic molecule. Our findings suggest that even in the situation where the electron dynamics are strongly correlated, features appearing in the time-resolved absorption cross section reflect the charge flow in the molecule in time and-when it is possible to make use of the element specificity of core-to-valence transitions-also in space. Compared to other more natural approaches to resolve electron dynamics with spatial resolution such as time-resolved x-ray scattering $[42,43]$ or electron diffraction [44], the ATA spectra allow easy and intuitive access to the electron dynamics. Moreover, we find that ATAS allows one to differentiate the dynamics associated with the population of different excitation classes not only when these dynamics are spatially separate but even when they affect the same atom. The surprisingly straightforward interpretability of the ATA spectra and the fact that ATAS is already feasible [7,8], makes ATAS, in our opinion, the most promising technique for monitoring correlated electron dynamics in complex molecular systems.

\section{ACKNOWLEDGMENT}

Funding by the excellence cluster "The Hamburg Centre for Ultrafast Imaging - Structure, Dynamics, and Control of Matter on the Atomic Scale" is gratefully acknowledged.

\section{APPENDIX A: VALENCE ELECTRON DYNAMICS: NUMERICAL APPROACH}

Here, we provide information concerning the computational method employed for the description of the valence electron dynamics investigated. In the situations considered, the initial state of the valence electrons of the dication is a two-hole configuration:

$$
|\psi(t=0)\rangle=c_{i} c_{j}\left|\Phi_{0}\right\rangle
$$

where $\left|\Phi_{0}\right\rangle$ denotes the Hartree-Fock ground state and $c_{i} c_{j}$ denote annihilation operators of Hartree-Fock spin orbitals. In the absence of the residual electron-electron interaction, these states would be eigenstates of the dicationic Hamiltonian so that the subsequent dynamic - if existing - is the result of the mutual interaction of the electrons captured in the dicationic molecule. Here, our starting point for the theoretical description and the numerical treatment of the valence electron dynamics that follow the preparation is the representation of the electronic wave function in terms of a configuration interaction expansion:

$$
\begin{aligned}
|\psi(t)\rangle= & \sum_{i<j} \alpha_{i, j}(t) c_{i} c_{j}\left|\Phi_{0}\right\rangle \\
& +\sum_{a} \sum_{i<j<k} \alpha_{i, j, k}^{a}(t) c_{a}^{\dagger} c_{i} c_{j} c_{k}\left|\Phi_{0}\right\rangle \ldots
\end{aligned}
$$

Here, ... stands for higher excitation classes such as the $4 \mathrm{H} 2 \mathrm{P}$ configurations (i.e., $c_{a}^{\dagger} c_{b}^{\dagger} c_{i} c_{j} c_{k} c_{l}\left|\Phi_{0}\right\rangle$ ), the $5 \mathrm{H} 3 \mathrm{P}$ configurations and so on until one ends up with the full-CI expansion. An approximation of this wave function by an expansion that is restricted to the two-hole configurations $c_{i} c_{j}\left|\Phi_{0}\right\rangle$ is possible when the valence electron dynamics are governed by the dynamics of the two induced electron holes.

$$
|\psi(t)\rangle \approx \sum_{i<j} \alpha_{i, j}(t) c_{i} c_{j}\left|\Phi_{0}\right\rangle .
$$

However, electron correlation and orbital relaxation can in general result in the promotion of electrons to initially unoccupied orbitals (virtual orbitals). As indicated in Eq. (A2), excitations of electrons to virtual orbitals can be taken into account by including systematically higher excitations classes such as the $3 \mathrm{H} 1 \mathrm{P}$ configurations $c_{i}^{\dagger} c_{j} c_{k} c_{l}\left|\Phi_{0}\right\rangle$, the $4 \mathrm{H} 2 \mathrm{P}$ configurations and so on until one ends up with a fully correlated description of the electron dynamics (full-CI). In this work, we restrict our considerations to those effects in which at maximum a single electron is promoted to the virtual orbitals. Accordingly, with the $2 \mathrm{H}$ configurations as reference states, we consider a time-dependent multireference configuration interaction singles expansion in the form of:

$$
\begin{aligned}
|\psi(t)\rangle \approx & \sum_{i<j} \alpha_{i, j}(t) c_{i} c_{j}\left|\Phi_{0}\right\rangle \\
& +\sum_{a} \sum_{i<j<k} \alpha_{i, j, k}^{a}(t) c_{a}^{\dagger} c_{i} c_{j} c_{k}\left|\Phi_{0}\right\rangle .
\end{aligned}
$$

With this, insight into final state correlation and relaxation can be obtained systematically by variation of the number of virtual orbitals taken into account. When no virtual orbitals are included in the calculation, an approximation in the form of Eq. (A3) is obtained whereas correlation and orbital relaxation effects associated with the promotion of a single electron to the virtual orbitals are taken into account when a finite number of virtual orbitals are included. In order to obtain approximations of the exact wave function in the form of the wave functions given in Eq. (A4), we propagate the initial state numerically exactly within the respective subspace. That is, the time-evolved states were determined by:

$$
|\psi(t)\rangle=e^{-i H t}|\psi(t=0)\rangle,
$$

where $H$ denotes the Hamiltonian matrix restricted to the respective subspace. When the initial state is represented in terms of the eigenvectors $|I\rangle$ of $H$ as denoted in Eq. (A6)

$$
|\psi(t=0)\rangle=\sum_{I} \alpha_{I}|I\rangle,
$$

the time-evolved state can be obtained by:

$$
|\psi(t)\rangle=\sum_{I} \alpha_{I} e^{-i E_{I} t}|I\rangle .
$$

Here, the coefficients $\alpha_{I}$ are the expansion coefficients of the initial state expanded in dicationic eigenstates $|I\rangle$ and $E_{I}$ are the corresponding energies. In the situations considered, the subspace dimensions are on the order of 200-10000 so that dicationic eigenstates can be obtained by full diagonalization 
of the Hamiltonian matrix using LAPACK [45]. The HartreeFock orbitals, the one-particle integrals of the kinetic energy and, the core potentials, which are needed for the calculation of the many-body Hamiltonian matrix elements, are taken from the quantum chemistry software MOLCAS [46]; the Coulomb matrix elements are obtained from the LIBINT library [47]. For the iodine atom and the carbon atom, we employed effective core potentials and associated basis sets from [48] (iodine), [49] (carbon), and, respectively, [50] (bromine) whereas for the hydrogen atoms 6-31 G basis sets were employed. The molecular geometries were determined by MOLCAS on the Hartree-Fock SCF wave function level.

\section{APPENDIX B: TIME-RESOLVED ATTOSECOND TRANSIENT ABSORPTION CROSS SECTION}

For a numerical determination of the time-resolved absorption cross section, we applied the theory of attosecond transient absorption spectroscopy developed in Ref. [35]. According to Ref. [35], for molecules that are oriented, the attosecond transient absorption cross section can be obtained in the short-pulse approximation and by using Beer's law by:

$$
\begin{aligned}
\sigma_{Z}(\omega, t)= & \frac{4 \pi \omega}{c} \operatorname{Im} \sum_{I, I^{\prime}} \alpha_{I}^{*} e^{i E_{I} t} \alpha_{I^{\prime}} e^{-i E_{I^{\prime}} t} \\
& \times \sum_{F}\langle I|\hat{Z}| F\rangle\left\langle F|\hat{Z}| I^{\prime}\right\rangle \\
& \times\left(\frac{1}{\tilde{E}_{F}-E_{I}-\omega}+\frac{1}{\tilde{E}_{F}^{*}-E_{I}^{\prime}+\omega}\right) .
\end{aligned}
$$

Here, $\hat{Z}$ is the component of the electric dipole operator along the laser polarization. In the situation where the molecules are completely unaligned (here, this situation is assumed), the cross section can be obtained by averaging over all possible orientations with respect to the laser polarization:

$$
\begin{aligned}
\sigma(\omega, t)= & \frac{\omega}{c} \operatorname{Im} \int_{0}^{\pi} \int_{0}^{2 \pi} d \phi d \theta \sin (\theta) \\
& \times \sum_{I, I^{\prime}} \alpha_{I}^{*} e^{i E_{I^{t}}} \alpha_{I^{\prime}} e^{-i E_{I^{\prime}} t} \\
& \times \sum_{F}\left\langle I\left|\hat{e}_{r}(\theta, \phi) \vec{R}\right| F\right\rangle\left\langle F\left|\hat{e}_{r}(\theta, \phi) \vec{R}\right| I^{\prime}\right\rangle \\
& \times\left(\frac{1}{\tilde{E}_{F}-E_{I}-\omega}+\frac{1}{\tilde{E}_{F}^{*}-E_{I}^{\prime}+\omega}\right) \\
= & \frac{\sigma_{X}(\omega, t)+\sigma_{Y}(\omega, t)+\sigma_{Z}(\omega, t)}{3} .
\end{aligned}
$$

Here, $\hat{e}_{r}(\theta, \phi)$ is the radial unit vector and $\vec{R}$ is the electric dipole operator. $\sigma_{X}, \sigma_{Y}, \sigma_{Z}$ are the cross sections, according to Eq. (B1), obtained for three orthogonal polarization directions.

The inner-shell vacancies decay within a few femtoseconds (see, for instance, Ref. [51]). For this reason, we assign the final states a finite lifetime $\frac{1}{\Gamma}$, which enters Eq. (B1) in form of complex final state energies $\tilde{E}_{F}=E_{F}-\frac{i \Gamma}{2}$. Here, we assumed a decay width of the inner-shell vacancies on the order of the atomic one, i.e., $\Gamma \sim 100 \mathrm{meV}$. For the discussion in the paper, this appears to be reasonable whereas for a direct comparison with experimental data more accurate values might be employed. The final core excited states are approximated as the valence excited states, i.e., they are approximated in terms of $2 \mathrm{H}$ configurations and $2 \mathrm{H}$ and $3 \mathrm{H} 1 \mathrm{P}$ configurations, respectively. This procedure allows us to describe in particular the dominant features in the cross section, which arise due to the presence of electron holes. To reduce the computational effort, the configuration subspaces are constructed such that at maximum a single hole is in an inner-shell orbital. Since in the situations considered, states with a double ionization potential (DIP) larger than $50 \mathrm{eV}$ are insignificantly populated (i.e., their populations are on the order of 0.001 and smaller), for a significant reduction in computation time, we restricted the sum in Eq. (B1) to

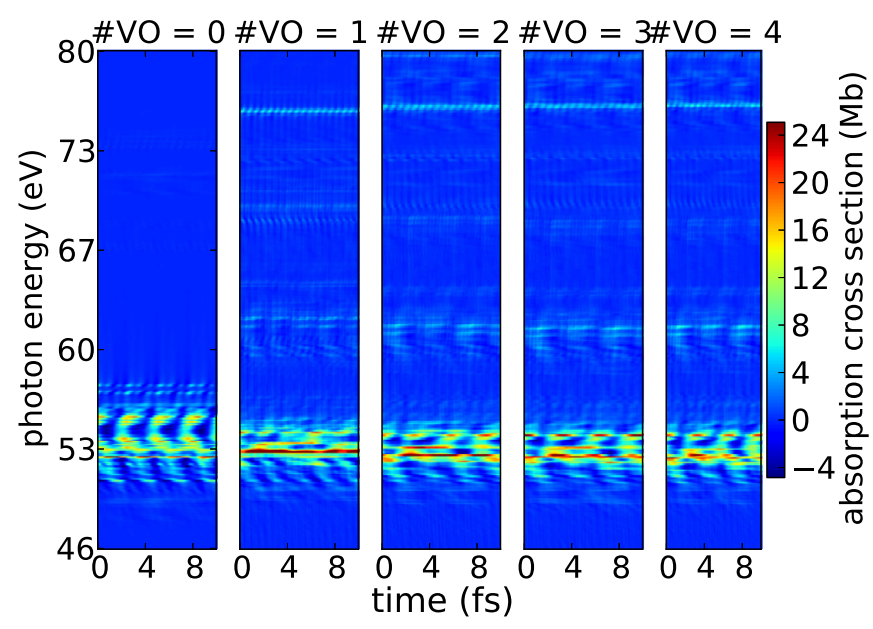

(a)

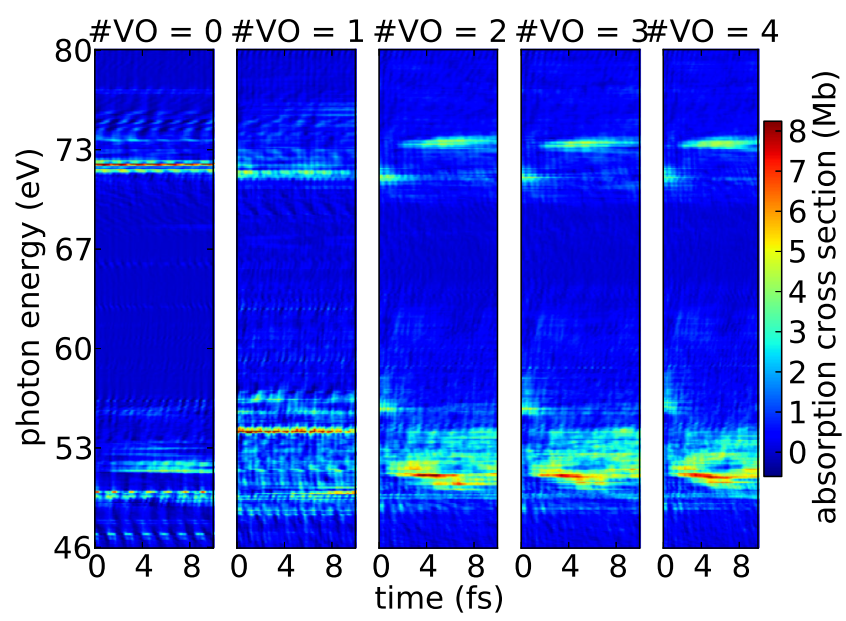

(b)

FIG. 8. The time-resolved absorption cross section in dependence of the number of virtual orbitals (VO) that are included in the calculation. Here, the situations are considered where (a) two electrons are removed from the HOMO and (b) two electrons are removed from the HOMO-5. Already by inclusion of two virtual orbitals, the features discussed arise. In the paper, we showed the time-resolved absorption cross section obtained from calculations in which four virtual orbitals were included. 
those states with a DIP lower than $50 \mathrm{eV}$. We also restrict the sum over the final states $|F\rangle$ to those states that are relevant in the spectral region considered (that is, for photon frequencies ranging from $\omega_{\min }=46 \mathrm{eV}$ to $\omega_{\max }=80 \mathrm{eV}$ ), i.e., those states $|F\rangle$ for which the condition $\omega_{\min }<\left|\tilde{E}_{F}-E_{I}\right|<$ $\omega_{\max }$ is fulfilled for all populated dicationic eigenstates $|I\rangle$. Furthermore, final states $|F\rangle$ for which the transition weights $\left|\alpha_{I}\right|^{2}|\langle I|Z| F\rangle|^{2}$ for all populated dicationic eigenstates $|I\rangle$ fall below $10^{-8}$ (in atomic units) are found to be irrelevant for the spectra determined and are consequently excluded from the calculation.

The features caused by particle-hole excitations discussed appear already when only two virtual orbitals are taken into account-increasing the number of included virtual orbitals from 2 to 4 reveals only small changes (see Fig. 8) but no new features arise.
[1] M. Hentschel, R. Kienberger, C. Spielmann, G. A. Reider, N. Milosevic, T. Brabec, P. Corkum, U. Heinzmann, M. Drescher, and F. Krausz, Nature (London) 414, 509 (2001).

[2] F. Krausz and M. Ivanov, Rev. Mod. Phys. 81, 163 (2009).

[3] F. Calegari, D. Ayuso, A. Trabattoni, L. Belshaw, S. De Camillis, S. Anumula, F. Frassetto, L. Poletto, A. Palacios, P. Decleva, J. B. Greenwood, F. Martín, and M. Nisoli, Science 346, 336 (2014).

[4] P. M. Kraus, B. Mignolet, D. Baykusheva, A. Rupenyan, L. Horný, E. F. Penka, G. Grassi, O. I. Tolstikhin, J. Schneider, F. Jensen, L. B. Madsen, A. D. Bandrauk, F. Remacle, and H. J. Wörner, Science 350, 790 (2015).

[5] L. Cederbaum and J. Zobeley, Chem. Phys. Lett. 307, 205 (1999).

[6] A. I. Kuleff and L. S. Cederbaum, J. Phys. B 47, 124002 (2014).

[7] E. Goulielmakis, Z.-H. Loh, A. Wirth, R. Santra, N. Rohringer, V. S. Yakovlev, S. Zherebtsov, T. Pfeifer, A. M. Azzeer, M. F. Kling, S. R. Leone, and F. Krausz, Nature (London) 466, 739 (2010).

[8] A. Wirth, R. Santra, and E. Goulielmakis, Chem. Phys. 414, 149 (2013).

[9] S. Pabst, M. Lein, and H. J. Wörner, Phys. Rev. A 93, 023412 (2016).

[10] S. R. Leone, C. W. McCurdy, J. Burgdorfer, L. S. Cederbaum, Z. Chang, N. Dudovich, J. Feist, C. H. Greene, M. Ivanov, R. Kienberger, U. Keller, M. F. Kling, Z.-H. Loh, T. Pfeifer, A. N. Pfeiffer, R. Santra, K. Schafer, A. Stolow, U. Thumm, and M. J. J. Vrakking, Nat. Photon. 8, 162 (2014).

[11] T. Kuś, B. Mignolet, R. D. Levine, and F. Remacle, J. Phys. Chem. A 117, 10513 (2013).

[12] B. Mignolet, R. D. Levine, and F. Remacle, Phys. Rev. A 86, 053429 (2012).

[13] B. Cooper and V. Averbukh, Phys. Rev. Lett. 111, 083004 (2013).

[14] J. Leeuwenburgh, B. Cooper, V. Averbukh, J. P. Marangos, and M. Ivanov, Phys. Rev. Lett. 111, 123002 (2013).

[15] D. Popova-Gorelova and R. Santra, Phys. Rev. B 91, 184303 (2015).

[16] A. D. Dutoi, K. Gokhberg, and L. S. Cederbaum, Phys. Rev. A 88, 013419 (2013).

[17] H. Wang, M. Chini, S. Chen, C.-H. Zhang, F. He, Y. Cheng, Y. Wu, U. Thumm, and Z. Chang, Phys. Rev. Lett. 105, 143002 (2010).

[18] M. Holler, F. Schapper, L. Gallmann, and U. Keller, Phys. Rev. Lett. 106, 123601 (2011).

[19] K. Ramasesha, S. R. Leone, and D. M. Neumark, Annu. Rev. Phys. Chem. 67, 41 (2016).

[20] S. Chen, M. J. Bell, A. R. Beck, H. Mashiko, M. Wu, A. N. Pfeiffer, M. B. Gaarde, D. M. Neumark, S. R. Leone, and K. J. Schafer, Phys. Rev. A 86, 063408 (2012).
[21] M. Chini, X. Wang, Y. Cheng, Y. Wu, D. Zhao, D. A. Telnov, S.-I. Chu, and Z. Chang, Sci. Rep. 3, 1105 (2013).

[22] C. Ott, A. Kaldun, L. Argenti, P. Raith, K. Meyer, M. Laux, Y. Zhang, A. Blattermann, S. Hagstotz, T. Ding, R. Heck, J. Madronero, F. Martín, and T. Pfeifer, Nature (London) 516, 374 (2014).

[23] S. G. Sayres, E. R. Hosler, and S. R. Leone, J. Phys. Chem. A 118, 8614 (2014)

[24] M. Schultze, E. M. Bothschafter, A. Sommer, S. Holzner, W. Schweinberger, M. Fiess, M. Hofstetter, R. Kienberger, V. Apalkov, V. S. Yakovlev, M. I. Stockman, and F. Krausz, Nature (London) 493, 75 (2013).

[25] M. Schultze, K. Ramasesha, C. Pemmaraju, S. Sato, D. Whitmore, A. Gandman, J. S. Prell, L. J. Borja, D. Prendergast, K. Yabana, D. M. Neumark, and S. R. Leone, Science 346, 1348 (2014).

[26] Z. Li, O. Vendrell, and R. Santra, Phys. Rev. Lett. 115, 143002 (2015).

[27] S. Lünnemann, A. I. Kuleff, and L. S. Cederbaum, J. Chem. Phys. 129, 104305 (2008).

[28] S. Lünnemann, A. I. Kuleff, and L. S. Cederbaum, J. Chem. Phys. 130, 154305 (2009).

[29] J. Breidbach and L. S. Cederbaum, J. Chem. Phys. 118, 3983 (2003).

[30] A. I. Kuleff, J. Breidbach, and L. S. Cederbaum, J. Chem. Phys. 123, 044111 (2005).

[31] H. Hennig, J. Breidbach, and L. S. Cederbaum, J. Phys. Chem. A 109, 409 (2005).

[32] A. I. Kuleff and L. S. Cederbaum, Chem. Phys. 338, 320 (2007).

[33] J. Breidbach and L. S. Cederbaum, J. Chem. Phys. 126, 034101 (2007).

[34] A. D. Dutoi and L. S. Cederbaum, Phys. Rev. A 90, 023414 (2014)

[35] R. Santra, V. S. Yakovlev, T. Pfeifer, and Z.-H. Loh, Phys. Rev. A 83, 033405 (2011).

[36] A. R. Attar, L. Piticco, and S. R. Leone, J. Chem. Phys. 141, 164308 (2014).

[37] G. Bruhn, E. R. Davidson, I. Mayer, and A. E. Clark, Int. J. Quantum Chem. 106, 2065 (2006).

[38] L. S. Cederbaum, J. Schirmer, W. Domcke, and W. von Niessen, J. Phys. B 10, L549 (1977).

[39] J. Schirmer, W. Domcke, L. S. Cederbaum, and W. von Niessen, J. Phys. B 11, 1901 (1978).

[40] E. M. Ohrendorf, F. Tarantelli, and L. S. Cederbaum, J. Chem. Phys. 92, 2984 (1990).

[41] J. E. Bækhøj, L. Yue, and L. B. Madsen, Phys. Rev. A 91, 043408 (2015). 
[42] G. Dixit, O. Vendrell, and R. Santra, Proc. Natl. Acad. Sci. USA 109, 11636 (2012).

[43] G. Dixit, J. M. Slowik, and R. Santra, Phys. Rev. A 89, 043409 (2014).

[44] H.-C. Shao and A. F. Starace, Phys. Rev. A 88, 062711 (2013).

[45] E. Anderson, Z. Bai, C. Bischof, S. Blackford, J. Demmel, J. Dongarra, J. Du Croz, A. Greenbaum, S. Hammarling, A. McKenney, and D. Sorensen, LAPACK Users' Guide, 3rd ed. (SIAM, Philadelphia, 1999).

[46] G. Karlström, R. Lindh, P.-Å. Malmqvist, B. O. Roos, U. Ryde, V. Veryazov, P.-O. Widmark, M. Cossi, B. Schimmelpfennig,
P. Neogrady, and L. Seijo, Comput. Mater. Sci. 28, 222 (2003), proceedings of the Symposium on Software Development for Process and Materials Design.

[47] E. F. Valeev, http://libint.valeyev.net/.

[48] K. A. Peterson, B. C. Shepler, D. Figgen, and H. Stoll, J. Phys. Chem. A 110, 13877 (2006).

[49] A. Bergner, M. Dolg, W. Küchle, H. Stoll, and H. Preuß, Mol. Phys. 80, 1431 (1993).

[50] K. A. Peterson, D. Figgen, E. Goll, H. Stoll, and M. Dolg, J. Chem. Phys. 119, 11113 (2003).

[51] J. N. Cutler, G. M. Bancroft, and K. H. Tan, J. Chem. Phys. 97, 7932 (1992). 